\title{
Harnessing the Power of Hospitalists in Operational Disaster Planning: COVID-19
}

\author{
Kasey Bowden, $\mathrm{NP}^{1,2}$, Ellen L. Burnham, $\mathrm{MD}^{2,3}$, Angela Keniston, $\mathrm{MSPH}^{1,2}$, \\ Dimitriy Levin, $M D^{1,2}$, Julia Limes, $M D^{1,2}$, Jason Persoff, $M D^{1,2}$, Lindsay Thurman, $M D^{1,2}$, \\ and Marisha Burden, $\mathrm{MD}^{1,2}$
}

'Division of Hospital Medicine, University of Colorado School of Medicine, Aurora, CO, USA; ${ }^{2}$ University of Colorado School of Medicine, Aurora, CO, USA; ${ }^{3}$ Division of Pulmonary Sciences and Critical Care Medicine, Aurora, CO, USA.

Hospitalists are well poised to serve in key leadership roles and in frontline care in particular when facing a pandemic such as the SARS-CoV-2 (COVID-19) infection. Much of the disaster planning in hospitals around the country addresses overcrowded emergency departments and decompressing these locations; however, in the case of COVID-19, intensive care units, emergency departments, and medical wards ran the risk of being overwhelmed by a large influx of patients needing high-level medical care. In a matter of days, our Division of Hospital Medicine, in partnership with our hospital, health system, and academic institution, was able to modify and deploy existing disaster plans to quickly care for an influx of medically complex patients. We describe a scaled approach to managing hospitalist clinical operations during the COVID-19 pandemic.

J Gen Intern Med 35(9):2732-7

DOI: $10.1007 / \mathrm{s} 11606-020-05952-6$

(c) Society of General Internal Medicine 2020

\section{INTRODUCTION}

On December 31, 2019, Chinese officials informed the World Health Organization of a "mysterious pneumonia" affecting 41 patients, all tied to the Huanan Wholesale Seafood Market. $^{1,2}$ Later identified as SARS-CoV-2 (COVID-19), COVID-19 would soon unfold into a pandemic impacting frontline responders across the world. ${ }^{3-5}$ Overburdened providers and healthcare systems would soon surface in China, Italy, and eventually the USA. ${ }^{6}$

Hospitalists have been at the forefront of the COVID-19 response, helping to lead frontline work, orchestrating complex and dynamic clinical operational plans, and partnering with clinicians and leaders from many different specialties. ${ }^{7-9}$ Much of the hospital disaster planning in the USA focuses on rapid intake of a large number of trauma patients in response to a time-limited event such as a natural disaster or a mass casualty incident. Prior pandemics such as the influenza H1N1 epidemic in 2009 directed attention to the importance of emergency preparedness for potentially large pandemics but failed to

Received April 21, 2020

Accepted May 29, 2020

Published online July 13, 2020 motivate sustained attention in the wake of a successful vaccine. In that context, COVID-19 was primed to overwhelm intensive care units, emergency departments, and medical wards.

Starting with preparedness models outlined by Persoff et al., ${ }^{10}$ Frank et al., ${ }^{8}$ the CHEST consensus statement, ${ }^{11,} 12$ and our existing disaster plans, we developed a modified framework for hospital operations management and staffing, anticipating a massive influx of acutely ill, medically complex, and highly contagious patients with COVID-19.

\section{THE FRAMEWORK: THE 4 C'S}

Using best practices in disaster planning, we developed a working framework for the management of the COVID-19 pandemic (Fig. 1).

Crucial to successfully addressing this challenge was collaboration: forming effective partnerships and information sharing across multiple groups.

Recognizing the imminent influx of a novel patient population, capacity building became an early priority - and became a guiding principle of the framework. In typical disaster planning, net new providers are brought in as quickly as possible; however, when caring for patients with a highly contagious virus, the potential risks associated with bringing multiple new providers into the hospital also needed to be balanced. Therefore, plans were devised to maximize the workforce already in the hospital, thereby limiting the need to call in additional providers until necessary.

Utilizing knowledge from Italy and China, it was anticipated that the workforce could also become overwhelmed by illness related to exposures from patient care or other caregiving issues indirectly related to the virus (e.g., child care due to school closures). ${ }^{13-16}$ Containment thus became a guiding principle to build systems that limited the number of providers exposed to highly contagious patient care environments. Backup call (i.e., jeopardy) became a last option to preserve the number of providers available to cover for those with illness or caregiving duties.

The last guiding principle was conscientiousness of resources (people, systems, and cost), knowing that any plan needed sustainability, particularly since successfully flattening 


\section{C Approach to Hospital Capacity Planning for COVID-19 Pandemic}

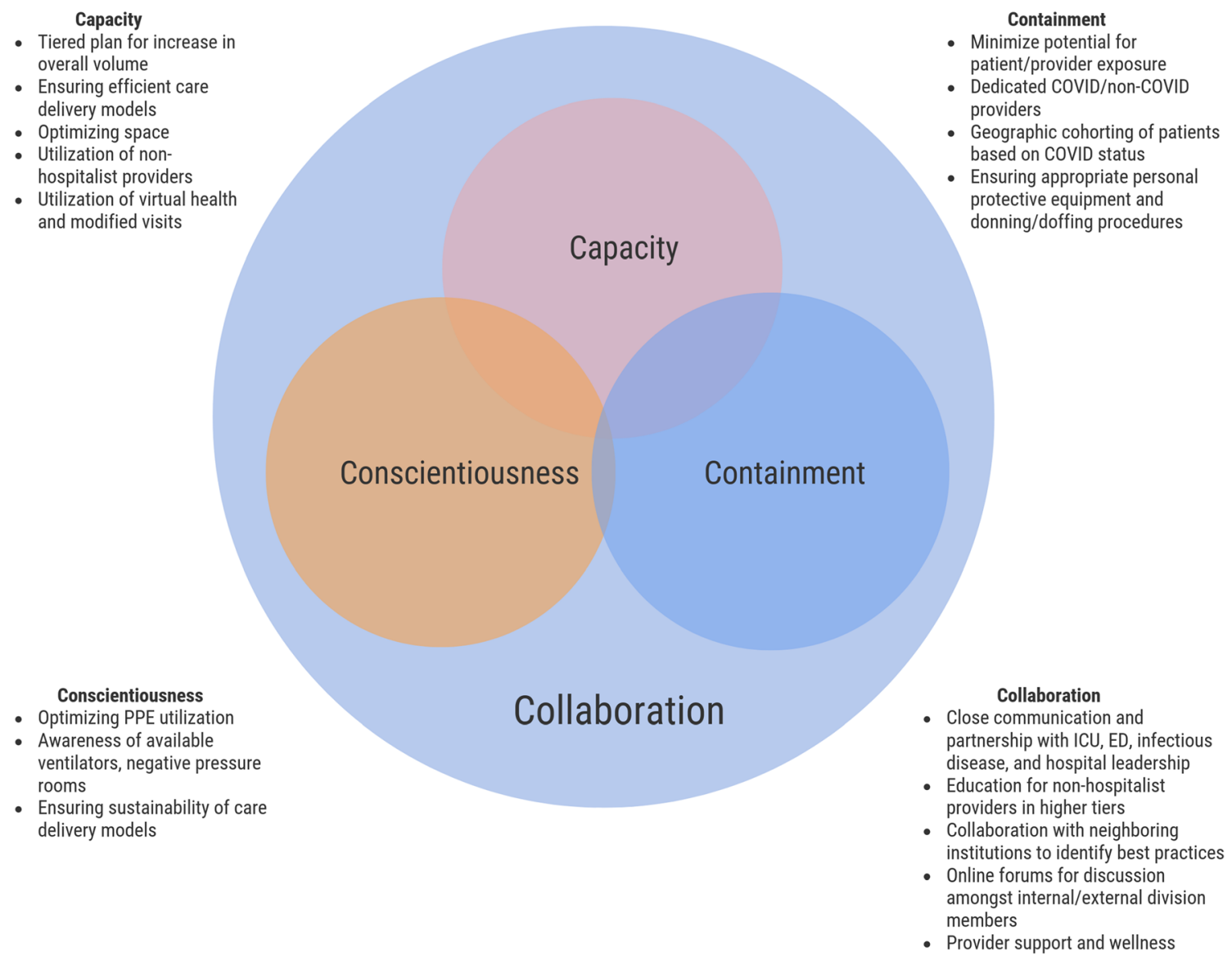

Fig. 1 Working framework for the management of the COVID-19 pandemic.

the curve would mean prolonging the plateau and thus prolongation of the surge of COVID-19 patients over time.

\section{A TIERED SYSTEM FOR THE MANAGEMENT OF COVID- 19 PANDEMIC (FIG. 2)}

Tier 1: Limit Adding New Providers to an Already Taxed System, Limit Unnecessary Exposure to Healthcare System, Convert Some Hospitalist Specialty Services (i.e., Consult, Oncology) to General Medical Teams, and Reserve Back Up/Jeopardy for Ill Providers and Those with Caregiving Duties

\section{Capacity Building}

In tier one, the capacity of all hospital medicine teams including teaching and non-teaching teams was increased above the usual census targets although still within the guidelines set forth by the Accreditation Council for Graduate Medical Education. Coverage for the afternoon hours to help with increased cross- cover was deployed as moonlighting. As elective surgeries were delayed or canceled, declines in patient volumes occurred, contributing to additional capacity on our internal medicine consult services and other specialty services such as hospitalist-led medical oncology teams where declines in patient volumes were also seen. Services such as Family Medicine and Pulmonary Critical Care became fluid partners with hospital medicine offering collective surge capacity with each team willing to flex to the other service(s) should the need arise.

A powerful and experienced advanced practice provider (APP) workforce was key to the Tier 1 plan allowing teams to flex to higher volumes while maximizing efficiency and patient throughput. Physician-APP teams at our institution typically manage around 14 to 16 patients together given the complexity of our patients. As part of our surge plan, our APPs could see patients independently (with physician back up as needed), pushing the team census to 20 patients (and up to 25) on each team if needed, while not requiring an individual clinician to care for overwhelming and potentially unsafe number of patients. Staffing protocols and clinical care algorithms for admission and discharge of patients with COVID19 also helped to facilitate these models which might not 


\section{Division of Hospital Medicine COVID Capacity Plan}

The 4 C's: Capacity building, containment (i.e. protecting our patients and our team), collaboration, and conscientious use of resources. This is a short term approach that helps to mitigate exponential increases in general medical patients and limits the unnecessary exposure to clinicians while preserving back up for clinician illness and care giving duties.

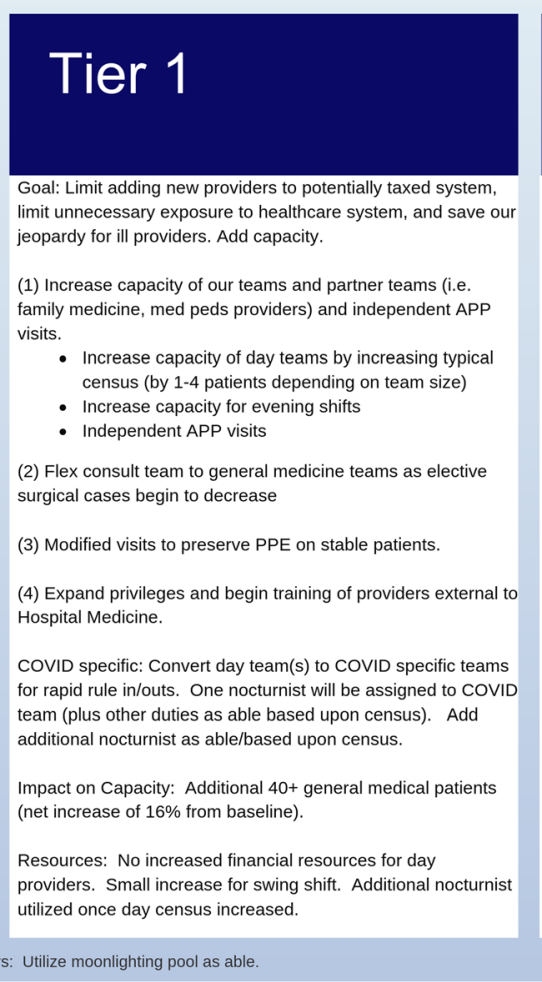

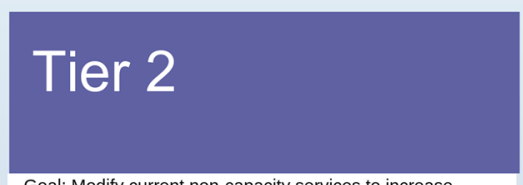

Goal: Modify current non-capacity services to increase overall capacity. Add additional day capacity to teams with innovative rounding models. Identify plan to offload critical care patients with expectation that ICU volumes will be high.

(1) Add nocturnist moonlighting as census rises.

(2) Admitting team converts to day capacity team.

(4) Utilize addiction medicine consult attending to add capacity.

(5) Continue to increase capacity of teams with APPs via independent visits.

(6) Activate subspecialty primary teams; activate subspecialty as ward team attendings. Utilize Department of Medicine faculty. (Tier 2,3). Attendings to be paired with learners or faculty. (Tier 2,3). Attendings to be paired
APPs who are familiar with inpatient EHR.

(7) Will flex hospitalists and/or ICU team depending on need Having daily calls with ICU team.

COVID specific: As COVID patient population continues to rise, will add additional geographically cohorted teams to care for COVID patients.

mpact on Capacity: Additional 40+ general medical patients (net increase of $45 \%$ from baseline) can be cared for depending on availability of other non-hospitalist providers.

Resources: Nocturnist cost, adding net new providers to system.

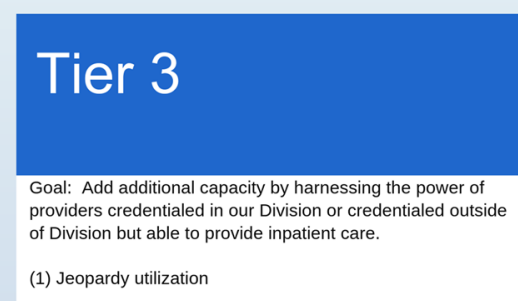

(3) Continue to activate subspecialty primary teams; activate subspeciality as ward team attendings

(4) Virtual health options for cross cover/alternative models of care; home cross cover to increase night capacity

(5) Physician Fellows or Physicians with UCH Affiliation not currently practicing at $\mathrm{UCH}$

(6) Cross hospital collaborations/flexing

COVID specific: Will continue to add teams as needed and will assess strategy continually.

Impact on Capacity: Additional 40+ general medical patients (net increase of $100 \%$ from baseline) can be cared for depending on availability of other non-hospitalist providers.

Resources: Adding additional net new providers to system

Fig. 2 The tiered system for the management of COVID-19 pandemic.

otherwise be possible with more typical medicine patients. Experienced hospitalist physicians and APPs were prioritized to these teams.

Because of the potential for a significant increase in admissions and cross-cover for evening and nights, times that are typically more sparsely staffed, additional providers were needed to cover those shifts when the patient numbers surged. An urgent moonlighting model harnessing APPs, physicians, and residents (when available) was built. To increase capacity while limiting the number of providers in the hospital, strategies were developed for remote patient care from home. For example, triagist work (admission flow and census management) was converted to a home-based service. Onsite modified visit protocols were developed for inpatient visits when a physical exam was not expected to change management. Providers were able to visit patients using HIPAA-compliant video chat platforms. Modified visits were deployed as a mechanism to decrease provider exposure to COVID-19 and to conserve personal protective equipment (PPE), in particular for consulting services and teaching teams to limit the number of providers entering the room.

\section{COVID-19-Specific Teams}

For disaster preparedness prior to COVID-19, some hospitalist providers had received specific training in the handling of special pathogens and donning/doffing procedures. While our team had a small number of highly trained hospitalists to work with special pathogens, it was apparent that, given the scale of the COVID-19 pandemic, those teams would be quickly overloaded. Thus, a hybrid approach was developed with dedicated COVID-19 teams staffed by a small group of providers that could be scaled as real-time learning and training occurred. Patients with COVID-19 or suspicion for COVID-19 were geographically cohorted, initially to one unit and one team, and then scaled as patient volumes increased. We partnered with our nursing colleagues to build these expert units and leveraged our electronic health record for enhanced communication strategies (i.e., secure chat platforms) to mitigate the constraints of PPE donning and doffing on communication.

\section{Impact}

The implementation of Tier 1 increased our team's ability to care for an additional $\sim 40$ general medical patients with a net 
increase in general medicine capacity of $16 \%$ without bringing in additional providers for day teams.

Tier 2: Modify Current Non-capacity Services Such as Admitter Shifts, Add Providers from Partner Groups (e.g., Subspecialists, Outpatient General Internists) and Flex In-house Teams as Needed (e.g., Intensivists, Hospitalists, Trauma Surgeons). Identify Plan to Offload Critical Care Patients with Expectation that Intensive Care Unit (ICU) Volumes Would Be High

\section{Capacity Building}

In Tier 2, much of the focus was on modifying existing services and flexing teams to the areas of greatest need. As ICU volumes grew, the intensive care unit volumes began to outgrow the floor volumes and hospitalist attendings and APPs were flexed to the ICU teams. Team members received just-in-time education with support from critical care experts. Because surgical volumes remained low, hospitalist consult teams were further consolidated and those providers were flexed to the ICU. Concurrently, Family Medicine team expanded their inpatient capacity by taking advantage of lower outpatient clinic volumes and partnering with Obstetrics and Gynecology $(\mathrm{Ob} / \mathrm{Gyn})$ to offload obstetric patients to $\mathrm{Ob} / \mathrm{Gyn}$ teams. Subspecialists and outpatient general internist volunteers were called in to supervise housestaff teams to allow additional hospitalists to function as ICU providers. Just-intime educational sessions were developed along with shared resources from groups across the country to train volunteer providers who had not recently worked in the inpatient setting.

\section{Impact}

Tier 2 allowed for a net capacity increase of at least 100 general medical patients (or a net increase of $45 \%$ of regular medicine volume from baseline) with added ability to provide care to critically ill COVID patients. As an example, each newly added provider could then care for upwards of 16 patients if needed and already existing consult services (and other specialty services) could then care for similar numbers given the substantial decrease in surgical volumes.

Tier 3: Increase Capacity by Tapping Providers Credentialed in Our Division or Credentialed Outside of the Division and Capable of Providing Inpatient Care

\section{Capacity Building}

In Tier 3, we planned to utilize jeopardy call (i.e., back up providers that we already had in place) plus an additional layers of volunteer clinicians within and external to the hospital system. APP providers from across the campus were surveyed and placed into a pool of providers who could be flexed to medical and intensive care unit services as needed. We had over 100 physician volunteers sign up from within our system and had additional APP providers external to our Division ready and willing to help cover our services. Because we have a centralized Office of Advanced Practice with close ties to our APPs across the health system, the office was able to orchestrate a robust back up pool quickly. While we did not have to implement Tier 3, based upon responses to our Tier 2 plan, we believe this represents a feasible surge model.

\section{Impact}

In all, the three tiers would allow for the care of at least 460 medically ill patients with an approximate $100 \%$ or greater increase in capacity from baseline to care for general medical patients.

Communication Strategies. The Hospital Medicine Clinical Operations leadership team built a forum to have daily huddles with hospital command center leadership, ICU leadership, and the designated COVID-19 team lead for the week. Staffing and flex plans were developed and deployed in real-time during the huddles and any specific issues that were raised were addressed. This allowed the clinicians who were on service to relay concerns with minimal interruption of clinical work. Calls with the Department of Medicine leadership including the Chair, Division heads for Infectious Disease, Hospital Medicine, and Pulmonary were held daily. Updates to the Division were consolidated into a nightly email to help streamline the flow of information, provide concise and consistent messaging, and limit the quantity of emails going out to the staff. Question and answer virtual townhalls were held with the entire Division twice a week. Hospital medicine and critical care teams held frequent calls each week with nursing leadership to plan for staffing needs and to anticipate how various units of the hospital along with nursing staffing as well as other ancillary staffing would be managed.

Data Strategies. The Division of Hospital Medicine Data and Analytics team developed three key reports: (1) a daily census report summarizing the number of patients with COVID by location and by need (ventilator utilization, extracorporeal membrane oxygenation); (2) an operational report with the number of admissions, discharges, length of stay, ICU transfers, and readmissions; (3) an EHR-based report with specific patient information for the leadership team. Reports (1) and (2) were sent to leadership daily.

Forecasting. To understand what scenarios would create high-capacity situations with more patients than patient to provider ratios could safely take care of, a forecasting tool was developed. Using this model, high-capacity situations with reduced ability to flex up were predicted to be most likely 
Table 1 Census situations and ability to flex staffing

\begin{tabular}{|c|c|c|c|c|c|c|}
\hline $\begin{array}{l}\text { Social distancing (i.e. } \\
\text { minimal } \\
\text { clinics/procedures) }\end{array}$ & $\begin{array}{l}\text { Predicted } \\
\text { ICU Census }\end{array}$ & $\begin{array}{l}\text { Predicted floor } \\
\text { COVID census }\end{array}$ & $\begin{array}{l}\text { Predicted floor } \\
\text { Non-COVID } \\
\text { census }\end{array}$ & $\begin{array}{c}\text { Predicted Impact on } \\
\text { overall census }\end{array}$ & $\begin{array}{l}\text { Hospital Medicine } \\
\text { need for external } \\
\text { support/volunteers }\end{array}$ & $\begin{array}{l}\text { Ability for other } \\
\text { teams to flex to } \\
\text { hospitalist/ICU }\end{array}$ \\
\hline \multicolumn{7}{|c|}{ COVID census increasing on floor and ICU; plus increase in non-COVID census } \\
\hline Yes & $\uparrow$ & $\uparrow$ & $\uparrow$ & $\uparrow \uparrow \uparrow \uparrow$ & $\uparrow \uparrow \uparrow \uparrow$ & $\uparrow$ \\
\hline No & $\uparrow$ & $\uparrow$ & $\uparrow$ & $\uparrow \uparrow \uparrow$ & $\uparrow \uparrow \uparrow$ & \\
\hline \multicolumn{7}{|c|}{ COVID census increasing on floor and ICU; non-COVID census decreased } \\
\hline Yes & $\uparrow$ & $\uparrow$ & $-/ \downarrow$ & $-/ \uparrow / \downarrow$ & $-/ \uparrow \uparrow / \downarrow \downarrow *$ & $\uparrow$ \\
\hline No & $\uparrow$ & $\uparrow$ & $-/ \downarrow$ & $-/ \uparrow / \downarrow$ & $-/ \uparrow \uparrow / \downarrow \downarrow^{*}$ & \\
\hline \multicolumn{7}{|c|}{ COVID ICU census trails and still increased, floor COVID census beginning to decrease; increase in non-COVID census (early) } \\
\hline Yes & $\uparrow$ & $-/ \downarrow$ & $\uparrow$ & $-/ \uparrow / \downarrow$ & $-/ \uparrow / \downarrow$ & $\uparrow$ \\
\hline No & $\uparrow$ & $-/ \downarrow$ & $\uparrow$ & $-/ \uparrow / \downarrow$ & $-/ \uparrow / \downarrow$ & $\nabla$ \\
\hline \multicolumn{7}{|c|}{ COVID ICU census trails and still increased, floor COVID census decreased; increase in non-COVID census (late) } \\
\hline Yes & $\bar{\uparrow}$ & $\downarrow$ & $\bar{\uparrow}$ & $\bar{\uparrow}$ & $-/ \uparrow$ & $\uparrow$ \\
\hline No & $\uparrow$ & $\downarrow$ & $\uparrow$ & $\uparrow$ & $-/ \uparrow$ & $\downarrow$ \\
\hline \multicolumn{7}{|c|}{ COVID census in ICU and floor decreasing, floor non-COVID census still down } \\
\hline Yes & $\downarrow$ & $\downarrow$ & $\downarrow$ & $\downarrow$ & $\downarrow$ & $n / a$ \\
\hline No & $\downarrow$ & $\downarrow$ & $\downarrow$ & $\downarrow$ & $\downarrow$ & $\mathrm{n} / \mathrm{a}$ \\
\hline \multicolumn{7}{|c|}{ COVID census in ICU and floor decreasing, floor non-COVID census increasing } \\
\hline Yes & $\downarrow$ & $\downarrow$ & $\uparrow$ & $-/ \uparrow$ & $-/ \uparrow$ & $-/ \uparrow$ \\
\hline No & $\downarrow$ & $\downarrow$ & $\uparrow$ & $-/ \uparrow$ & $-/ \uparrow$ & $-1 \downarrow$ \\
\hline
\end{tabular}

Green $=$ feasible staffing

Red $=$ staffing model at risk given decreased availability of clinicians

- = neither increased or decreased

*Dependent on how significant of a decrease of non-COVID patients occurs

to occur when hospital and clinic operations began to return to normal while the COVID-19 patient volumes were still high (Table 1).

Sustainability. This plan is meant to help guide when a shortterm, unexpected need arises and is not meant to replace appropriate medium to longer term staffing solutions.

As with any surge plan, ensuring sustainability is key. Our model allows for a temporary increased workload in a pandemic situation where patients have a single uniting illness and the surge is expected to be short-term. With reduced volumes of other patients in our healthcare system, we were able to utilize space, providers, and ancillary services usually dedicated to other forms of medical/surgical care.

Generalizability. We work at a large, urban academic hospital with multiple hospitals within the system. While our plan has seemingly been successful at our institution, it is likely modifications would need to be considered at other sites and thus, this report hopefully serves as a guide/menu of options for hospitals and their hospital systems to consider.

\section{SUMMARY}

Hospitalist groups across the country have assumed numerous roles during the COVID-19 pandemic, from frontline providers to operational leaders for their groups, hospitals, and health systems. We have provided a framework that maximizes providers within the hospital while also taking advantage of a wide array of the clinician workforce, minimizing exposures as well as maximizing clinician groups who might not otherwise be as involved in inpatient care.

Corresponding Author: Marisha Burden, MD; Division of Hospital Medicine, University of Colorado School of Medicine, Aurora, CO, USA (e-mail: marisha.burden@cuanschutz.edu). 


\section{Compliance with Ethical Standards:}

Conflict of Interest: The authors declare that they do not have a conflict of interest.

\section{REFERENCES}

1. Secon H, Woodward A, Mosher D. A comprehensive timeline of the new coronavirus pandemic, from China's first COVID-19 case to the present. Business Insider. https://www.businessinsider.com/coronavirus-pandemic-timeline-history-major-events-2020-3. Accessed April 16, 2020.

2. Wu F, Zhao S, Yu B, et al. A new coronavirus associated with human respiratory disease in China. Nature 2020;579:265-9.

3. Fauci AS, Lane HC, Redfield RR. Covid-19 - navigating the uncharted. N Engl J Med 2020;382:1268-9.

4. Holshue ML, DeBolt C, Lindquist S, et al. First case of 2019 novel coronavirus in the United States. N Engl J Med 2020;382:929-36.

5. Zhu N, Zhang $\mathbf{D}$, Wang $\mathbf{W}$, et al. A novel coronavirus from patients with pneumonia in China, 2019. N Engl J Med 2020;382:727-33.

6. Faccincani R, Pascucci F, Lennquist $\mathbf{S}$. How to surge to face SARS-CoV2 outbreak. Lessons learned from Lumbardy, Italy. Disaster Med Public Health Prep 2020:1-7.

7. Katie A Meier, MD, Karen E Jerardi, MD, MEd, Angela M Statile, MD, MEd, Samir S Shah, MD, MSCE, MHM, Pediatric hospital medicine management, staffing, and well-being in the face of COVID-19. Published online first April 14, 2020. DOI: 10.12788/jhm.3435close.
8. Frank MG, Croyle C, Beitscher A, Price C. The role of hospitalists in biocontainment units: a perspective. J Hosp Med 2020;15:E1-E3.

9. Garg M, Wray CM. Hospital medicine management in the time of COVID19: preparing for a sprint and a marathon. J Hosp Med 2020;15:305-7.

10. Persoff $\mathbf{J}$, Ornoff $\mathbf{D}$, Little C. The role of hospital medicine in emergency preparedness: a framework for hospitalist leadership in disaster preparedness, response, and recovery. J Hosp Med 2018;13:713-8.

11. Einav S, Hick JL, Hanfling $\mathbf{D}$, et al. Surge capacity logistics: care of the critically ill and injured during pandemics and disasters: CHEST consensus statement. Chest 2014; 146:e17S-43S.

12. Hick JL, Einav S, Hanfling $\mathbf{D}$, et al. Surge capacity principles: care of the critically ill and injured during pandemics and disasters: CHEST consensus statement. Chest 2014;146:e1S-e16S.

13. Bayham J, Fenichel EP. Impact of school closures for COVID-19 on the US health-care workforce and net mortality: a modelling study. Lancet Public Health 2020.

14. Hsin DH, Macer DR. Heroes of SARS: professional roles and ethics of health care workers. J Infect 2004;49:210-5.

15. Ran $\mathbf{L}$, Chen $\mathbf{X}$, Wang $\mathbf{Y}$, Wu W, Zhang $\mathbf{L}$, Tan $\mathbf{X}$. Risk factors of healthcare workers with corona virus disease 2019: a retrospective cohort study in a designated hospital of Wuhan in China. Clin Infect Dis 2020.

16. Wang J, Zhou M, Liu F. Reasons for healthcare workers becoming infected with novel coronavirus disease 2019 (COVID-19) in China. J Hosp Infect 2020.

Publisher's Note: Springer Nature remains neutral with regard to jurisdictional claims in published maps and institutional affiliations. 